\title{
The effect of pyridostigmine on bispectral index during recovery from sevoflurane anesthesia
}

\author{
Seol-Joo Jeong, Jong In Han, Hee-Jung Baik, Heeseung Lee, Guie Yong Lee, and Jong-Hak Kim \\ Department of Anesthesiology and Pain Medicine, School of Medicine, Ewha Womans University, Seoul, Korea
}

Background: There have been some conflicting reports showing that muscle relaxants and anticholinesterases affect the level of the bispectral index (BIS). The purpose of this study was to investigate whether pyridostigmine affects the level of the BIS during recovery from sevoflurane anesthesia.

Methods: Fifty-two adult patients scheduled for laparoscopic cholecystectomy and laparoscopic appendectomy. Anesthesia was induced with thiopental $4 \mathrm{mg} / \mathrm{kg}$ and rocuronium $0.6 \mathrm{mg} / \mathrm{kg}$. The lung was mechanically ventilated with $1-3$ vol\% sevoflurane, $50 \%$ oxygen and $50 \%$ nitrous oxide. After a specimen was removed, the sevoflurane concentration was maintained at 1.5 vol\%. When skin closure began, sevoflurane was stopped; however, $50 \%$ oxygen and 50\% nitrous oxide were maintained. The patients then received either (1) a group that received an injection of glycopyrrolate $0.04 \mathrm{mg} / \mathrm{kg}$ and pyridostigmine $0.2 \mathrm{mg} / \mathrm{kg}$ (reverse (R) group, $\mathrm{n}=26$ ) or (2) a group that received normal saline (control (C) group, $n=26$ ). Group assignment was random. Pyridostigmine, a reversible cholinesterase inhibitor, is a parasympathomimetic. End-tidal sevoflurane concentration, train of four (TOF) ratio, bispectral index (BIS), blood pressure and heart rate were measured from the end of the operation to 15 min after inject of pyridostigmine or placebo.

Results: There were no significant between group differences in the time dependent decrease in end-tidal sevoflurane concentration $(\mathrm{P}=0.0642)$. There were significant differences between the two groups for the time course for increases in the TOF value $(\mathrm{P}<0.0001)$. There were significant differences between the two groups for the time course for increases in the BIS value $(\mathrm{P}=0.0107)$. There were no significant differences in the mean BIS value up to 10 minutes after administering drug, but 15 minutes after administrating the reverse drug or the control drug, the BIS value showed significantly different BIS values: 68.2 \pm 6.2 (Group R) and 63.2 \pm 6.2 (Group C) $(\mathrm{P}=0.0058$ ).

Conclusions: The finding that pyridostigmine increases TOF and BIS suggests that pyridostigmine may enhance recovery during recovery from sevoflurane anesthesia. (Korean J Anesthesiol 2011; 61: 460-464)

Key Words: Bispectral index, Pyridostigmine, Sevoflurane, TOF ratio.

Received: January 31, 2011. Revised: March 9, 2011. Accepted: March 15, 2011.

Corresponding author: Jong In Han, M.D., Department of Anesthesiology and Pain Medicine, School of Medicine, Ewha Womans University, 911-1, Mok5-dong, Yangcheon-gu, Seoul 157-710, Korea. Tel: 82-2-2650-5559, Fax: 82-2-2655-2924, E-mail: hanji@ewha.ac.kr

(c) This is an open-access article distributed under the terms of the Creative Commons Attribution Non-Commercial License (http:// creativecommons.org/licenses/by-nc/3.0/), which permits unrestricted non-commercial use, distribution, and reproduction in any medium, provided the original work is properly cited. 


\section{Introduction}

The bispectral index (BIS) analyzes the electroencephalogram (EEG) to quantify it into measurable values and is used as a brain function monitor that measures the level of a patient's consciousness. Therefore, it can provide objective measurements of narcotic effects from anesthesia [1,2]. BIS is clinically helpful because it can ensure the right amount of anesthesia is used and prevent the danger of complications from over-dosage or arousal from under-dosage during an operation [3].

Muscle relaxants can decrease the activity of an electromyogram (EMG) affecting BIS [4]. According to afferentation theory, which argues that stretch receptors of the muscles stimulate the brain's awaking center, muscle relaxants block the muscle stretch receptors and decreases stimuli that awaken the brain and thus exhibit sedative effects [5]. There are reports that cholinesterase inhibitors cause a significant increase in the BIS value [6], but there are also contradicting reports [7]. Hence, there is a need to resolve this controversy.

Accordingly, the aim of this research was to determine the effects of pyridostigmine, an anticholinesterase, on recovery from anesthesia, by observing changes in BIS as a function of the degree of recovery from muscle relaxants when the end tidal concentration of sevoflurane is at $0.2 \mathrm{vol} \%$.

\section{Materials and Methods}

After obtaining approval of our Institutional Review Board and written informed consent from the patients, we investigated 52 patients, aged 16-65 years, with American Society of Anesthesiologists (ASA) Physical status I and II who were scheduled for laparoscopic cholecystectomy or appendectomy under general anesthesia. They were randomly divided into two groups of 26 patients each: one with and one without pyridostigmine. We excluded patients with a past history of illness of the heart, liver, kidney, or central nervous system, those who took sedatives, antidepressants, or sleeping pills, and those who were pregnant.

Midazolam $(0.05 \mathrm{mg} / \mathrm{kg})$ and $0.5 \mathrm{mg}$ of atropine were injected intramuscularly 30 minutes prior to the induction of anesthesia. After arrival in the operating room, the patients were monitored using an electrocardiograph, non-invasive blood pressure monitor, and pulse oximeter. A BIS sensor (BISquatro $^{\text {TM }}$, Aspect ${ }^{\circledR}$ Medical Systems, Inc., USA) was attached and measurements were taken when the signal quality index (SQI) was above 95. Muscle relaxation was monitored using an electrical stimulator (Relaxograph ${ }^{\circledR}$, Datex Relaxograph, Finland) by applying train of four (TOF) on the wrist's ulnar nerve and measuring the contraction of the adductor pollicis muscle.
Anesthesia was induced with $4 \mathrm{mg} / \mathrm{kg}$ of thiopental and $2 \mathrm{ug} /$ $\mathrm{kg}$ of fentanyl, and $0.6 \mathrm{mg} / \mathrm{kg}$ of rocuronium was administered as a muscle relaxant. Then, anesthesia was maintained with $1-3 \mathrm{vol} \%$ of sevoflurane and $1.5 \mathrm{~L} / \mathrm{min}$ each of nitrous oxide and oxygen. Temperature was maintained above $35^{\circ} \mathrm{C}$, and the respiration rate and tidal volume were regulated to maintain minute volumes of carbon dioxide tension at $30-35 \mathrm{mmHg}$. When the TOF number rose above 4 and showed train of four ratios, $0.2 \mathrm{mg} / \mathrm{kg}$ of rocuronium was administered. After the specimen was removed, the concentration of sevoflurane was maintained at 1.5 vol\% in both groups.

When skin closure began, sevoflurane was stopped but nitrous oxide and oxygen were continually maintained at $1.5 \mathrm{~L} /$ min for each. After surgery, a closed envelope with the group written inside was randomly selected prior to administering drugs. The Reverse (R) Group $(\mathrm{n}=26)$ received $0.04 \mathrm{mg} / \mathrm{kg}$ of glycopyrrolate and $0.2 \mathrm{mg} / \mathrm{kg}$ of pyridostigmine. The Control (C) group $(n=26)$ received an equal volume of normal saline. The end-tidal sevoflurane concentration, TOF ratio, and BIS were measured immediately after skin closure, and 5, 10, and 15 minutes after skin closure. To reduce the occurrence of changes from external stimuli, the operating room was maintained as a quiet environment, and no stimuli was given. Cases of awakening or coughing during the measurements were excluded from the study.

The number of subjects for each group was based on research by Vasella et al. [6]. In the power calculations, we used 0.05 as a criterion for statistical significance (alpha) and a power of $80 \%$ as a level of power deemed sufficient. The minimum sample size turned out to be 23 per group so the study was done using 26 subjects in each group.

All measurements were expressed as the mean and standard deviation, and an unpaired t test was done to compare groups using SPSS (version 17.0, SPSS Inc., Chicago, IL, USA). Chisquare tests were done for gender comparisons between the groups and repeated measures ANOVA was done for each group's changes in measurements as a function of time. $\mathrm{P}<0.05$ was defined as statistically significant.

\section{Results}

There were no between group differences in sex, age, height, weight, and time under anesthesia, but the operating time was significantly longer in the control group $(\mathrm{P}=0.0335$, Table 1$)$.

There were no significant between group differences in the decrease in end-tidal sevoflurane concentration $(\mathrm{P}=0.0642$, Table 2).

There were significant differences between the two groups for the time course for increases in the TOF value $(\mathrm{P}<0.0001$, Table 2). 
Table1. Demographic Data

\begin{tabular}{lccc}
\hline & Reverse group $(\mathrm{n}=26)$ & Control group $(\mathrm{n}=26)$ & P \\
\hline Sex (M/F) & $8 / 18$ & $8 / 18$ & 0.000 \\
Age (yr) & $38.0 \pm 13.9$ & $37.3 \pm 12.5$ & 0.851 \\
Height (cm) & $164.5 \pm 8.6$ & $162.1 \pm 5.7$ & 0.247 \\
Weight (kg) & $60.8 \pm 11.4$ & $63.1 \pm 9.7$ & 0.449 \\
Operation time (min) & $65.8 \pm 23.5$ & $85.4 \pm 39.0$ & $0.034^{*}$ \\
Anesthesia time (min) & $97.9 \pm 27.9$ & $116.6 \pm 42.5$ & 0.068 \\
\hline
\end{tabular}

Data are presented as mean \pm SD or number of cases. Reverse group: those who received pyridostigmine-glycopyrrolate, Control group: those who received saline as a placebo. $* \mathrm{P}<0.05$ vs placebo group.

Table 2. Changes in Sevoflurane Concentration (vol\%), TOF and BIS during the Study Period

\begin{tabular}{llccc}
\hline & & Reverse group & Control group & P \\
\hline \multirow{2}{*}{ Sevo (\%) } & Skin closure & $1.41 \pm 0.08$ & $1.43 \pm 0.08$ & 0.3073 \\
& 5 min & $0.45 \pm 0.07$ & $0.50 \pm 0.08$ & 0.0679 \\
& 10 min & $0.26 \pm 0.05$ & $0.33 \pm 0.08$ & 0.0007 \\
TOF & 15 min & $0.18 \pm 0.05$ & $0.22 \pm 0.08$ & 0.0562 \\
& Skin closure & $27.6 \pm 10.9$ & $27.5 \pm 11.7$ & 0.9805 \\
& 5 min & $57.2 \pm 17.8$ & $41.7 \pm 19.9$ & $0.0047^{*}$ \\
BIS & 10 min & $83.2 \pm 16.4$ & $56.2 \pm 21.2$ & $<0.0001^{*}$ \\
& 15 min & $90.3 \pm 12.6$ & $67.1 \pm 21.3$ & $<0.0001^{*}$ \\
& Skin closure & $45.2 \pm 5.5$ & $44.3 \pm 5.0$ & 0.5257 \\
& 5 min & $54.7 \pm 6.8$ & $53.9 \pm 7.2$ & 0.7080 \\
& 10 min & $62.4 \pm 5.4$ & $59.8 \pm 6.5$ & 0.1200 \\
& 15 min & $68.2 \pm 6.2$ & $63.2 \pm 6.2$ & $0.0058^{*}$ \\
\hline
\end{tabular}

Sevo (\%), TOF and BIS are presented as mean \pm SD. Sevo (\%): end-tidal sevoflurane concentration, Reverse group: those who received pyridostigmine-glycopyrrolate, Control group: those who received saline as a placebo, ${ }^{*} \mathrm{P}<0.05$ vs placebo group.

There were significant differences between the two groups for the time course for increases in the BIS value $(\mathrm{P}=0.0107$, Table 2).

There were no significant differences in the mean BIS value up to 10 minutes after administering drugs, but 15 minutes after administrating drugs (the reverse drug or the control drug), the BIS value showed a statistically significant difference with BIS values of $68.2 \pm 6.2$ (Group R) and $63.2 \pm 6.2$ (Group C) $(\mathrm{P}=$ 0.0058, Table 2).

Three patients in each group who woke up or coughed during the experiment were excluded from the study.

\section{Discussion}

The aim of this study was to determine whether the administration of pyridostigmine during recovery from sevoflurane anesthesia affects BIS values. There were no differences between the two groups up to 10 minutes after administrating the reverse or control drugs, but there were statistically significant differences 15 minutes after administrating the reverse drug at time at which the maximum effect of pyridostigmine is anticipated. Therefore, the study showed meaningful results. However, the values were 68.2 in the reverse group and 63.2 in the control group; so both showed a deep state of sedation. This is a small difference compared to the wide difference in TOF ratios.

BIS is used to measure consciousness during surgery and the degree of sedation to manage anesthesia in patients undergoing surgery. But it is measured by analyzing the EEG, so it can be influenced by any factors that affect or interfere with the activation of the EEG. In addition, notable activation of the EMG interferes with the collection of EEG signals. This confounds the calculation of the BIS value because although the patient is sedated, there could be activity due to selfrespiration. Generally, EEG signals exist in the $0.5-30 \mathrm{~Hz}$ range and EMG signals in the $30-300 \mathrm{~Hz}$ range; so EMG signals can influence the BIS, which analyzes EEG signals up to $47 \mathrm{~Hz}$ [8, 9]. However, when the patient is deeply sedated, as in general anesthesia, EMG activity does not affect the BIS. Greif et al. [7] reported that the BIS for EMG activity was not decreased by the administration of mivacurium when the BIS was about 40 and anesthesia was induced by propofol and the EMG activity was low (27 $\pm 1 \mathrm{~dB}$ ). However, Vasella et al. [6] reported that administration of neostigmine in propofol-remifentanil induced anesthesia changed the depth of the anesthesia and accelerated the recovery. In addition, Liu et al. [4] reported that if muscle relaxants were administered when anesthesia was shallow (with a BIS value higher than 72 and EMG activity at 
approximately $38 \mathrm{~dB}$ ), EMG activity decreased and BIS values decreased significantly. Messner et al. [10] reported a study in which succinylcholine was administered to volunteers who were awake. Five seconds later, EMG activity disappeared and the BIS decreased until it showed a deep anesthetic state (BIS 33). EMG activity reappeared and the BIS recovered to normal 5 minutes later.

In our study, 15 minutes after skin closure, EMG activity was maintained at a low state (below 1 bar; $30 \mathrm{~dB}$ ) when the endtidal sevoflurane concentration was maintained at $0.2 \mathrm{vol} \%$ and the BIS value was maintained at $60-70$. Therefore, it seems that the EMG activity did not interfere with the BIS. Thus, it can be said that in an anesthetic state, such as in our study, with a BIS value of $40-70$, administration of pyridostigmine does not increase EMG activity or BIS; but it does affect the BIS when the depth of anesthesia becomes shallower.

Another reason why muscle relaxants influence the BIS can be due to afferentation theory, which states that stretch receptors of muscles stimulate the awakening center of the brain and show awakening effects [5]. When muscle relaxation is achieved, a sedation effect appears because the stretch receptors of the muscles are blocked, which reduces the stimulus that awakens the brain. In a canine experiment, when a noxious stimulus was given during shallow halothane anesthesia, dogs who were not IV injected with pancuronium showed muscle movement and increased cerebral responses through afferent acceptors. But in dogs who were IV injected with pancuronium, the stimulus from the afferent acceptors disappeared and cerebral responses decreased. Forbes et al. [11] reported a $25 \%$ decrease of the halothane MAC in patient groups administered pancuronium. Schwartz et al. [12] showed that muscle relaxants increase the depth of anesthesia and reduce the amount of isoflurane required. Their study was a canine experiment in which pancuronium was administered in during isoflurane induced anesthesia. Pancuromium increased the effect of the anesthesia and increased the generation of burst suppression in the brain waves. Hodgson and Liu [13] reported that when lidocaine was administered through the epidural space, it decreased, by about $34 \%$, the amount of sevoflurane required to maintain sufficient anesthesia at a BIS lower than 50 , which was due to spinal deafferentation.

In our study, at 15 minutes, which is the time of pyridostigmine's maximum effect, the mean BIS value showed a statistically significant difference in which Groups R and C were 68.2 and 63.2, respectively. The BIS is thought to increase because the brain is awakened by the increased stimulus from the recovered muscle stretch receptors as a result of the administration of pyridostigmine.

There were no differences in clinical symptoms of waking or coughing between the groups and this was thought to be because both groups showed deep sedation as indicated by BIS values of 68.2 and 63.2. After the specimen was removed, the sevoflurane concentration was maintained at $1.5 \mathrm{vol} \%$; it was stopped after skin closure. Fifteen minutes after skin closure, the sevoflurane concentration was maintained at 0.2 vol\% with nitrous oxide continually maintained at $50 \%$ so the MAC value was maintained at above 0.6 , and there was no external stimulus.

Kim et al. [14] reported that stopping the administration of nitrous oxide when anesthesia was maintained with 2.5 vol\% sevoflurane, nitrous oxide ( $2 \mathrm{~L} / \mathrm{min})$, and oxygen $(2 \mathrm{~L} / \mathrm{min})$ may not cause hemodynamic changes but can increase the BIS and even rapidly increase the BIS depending on the strength of the stimulus. Barr et al. [15] reported that when nitrous oxide up to $70 \%$ was used as a single anesthetic, it did not influence the BIS in a study done on healthy volunteers. It caused loss of consciousness, but did not affect the BIS. However, the addition of $1.2 \mathrm{vol} \%$ of sevoflurane for a short period of time induced loss of consciousness and decreased the BIS. Therefore, in our study, nitrous oxide was maintained at the same concentration throughout the experiment so its affect on BIS was consistent, and thus, this study could focus on changes in the BIS induced by cholinesterase inhibitors.

The limitations of our study were as follows. The three excluded patients in each group who had awakened and coughed showed a rapid increase in EMG and BIS values. For accurate comparison between the two groups, these patients should be included. However, when there is an increase in the EMG value, the EMG value itself affects the BIS. So, it may be difficult to reflect the intent of this study. In addition, BIS values are quantifications of analyzed measurements that were taken from the previous minute because BIS analysis gathers data and goes through the process of time domain analysis, frequency domain analysis, and bispectral domain analysis and uses advanced multivariate statistical data analysis to display the components of the multiple EEG signal processing into a single variate index which all takes about a minute for the whole process [1]. Therefore, the BIS values for 15 minutes after administration of pyridostigmine is actually the analysis of EEG wave values 14 minutes after the administration of pyridostigmine, and since the maximum effect time of pyridostigmine is 15 minutes after its administration, the BIS value for 16 minutes may have been higher.

In conclusion, during recovery from sevoflurane with an end-tidal sevoflurane concentration of $0.2 \mathrm{vol} \%$, pyridostigmine decreased the recovery time of TOF and increased BIS values. This seems to be due to recovery from the sedative effect of the stretch receptors of the muscles. The lack of a clinical difference is thought to be due to the anesthetic influence from the remaining sevoflurane and nitrous oxide. 


\section{References}

1. Rampil IJ. A primer for EEG signal processing in anesthesia. Anesthesiology 1998; 89: 980-1002.

2. Glass PS, Bloom M, Kearse L, Rosow C, Sebel P, Manberg P. Bispectral analysis measures sedation and memory effects of propofol, midazolam, isoflurane, and alfentanil in healthy volunteers. Anesthesiology 1997; 86: 836-47.

3. Ekman A, Lindholm ML, Lennmarken C, Sandin R. Reduction in the incidence of awareness using BIS monitoring. Acta Anaesthesiol Scand 2004; 48: 20-6.

4. Liu N, Chazot T, Huybrechts I, Law-Koune JD, Barvais L, Fischler M. The influence of a muscle relaxant bolus on bispectral and datexohmeda entropy values during propofol-remifentanil induced loss of consciousness. Anesth Analg 2005; 101: 1713-8.

5. Lanier WL, Iaizzo PA, Milde JH, Sharbrough FW. The cerebral and systemic effects of movement in response to a noxious stimulus in lightly anesthetized dogs. Possible modulation of cerebral function by muscle afferents. Anesthesiology 1994; 80: 392-401.

6. Vasella FC, Frascarolo P, Spahn DR, Magnusson L. Antagonism of neuromuscular blockade but not muscle relaxation affects depth of anaesthesia. Br J Anaesth 2005; 94: 742-7.

7. Greif R, Greenwald S, Schweitzer E, Laciny S, Rajek A, Caldwell JE, et al. Muscle relaxation does not alter hypnotic level during propofol anesthesia. Anesth Analg 2002; 94: 604-8.

8. Johansen JW, Sebel PS. Development and clinical application of electroencephalographic bispectrum monitoring. Anesthesiology 2000; 93: 1336-44.

9. Johansen JW. Update on bispectral index monitoring. Best Pract Res Clin Anaesthesiol 2006; 20: 81-99.

10. Messner M, Beese U, Romstöck J, Dinkel M, Tschaikowsky K. The bispectral index declines during neuromuscular block in fully awake persons. Anesth Analg 2003; 97: 488-91.

11. Forbes AR, Cohen NH, Eger EI 2nd. Pancuronium reduces halothane requirement in man. Anesth Analg 1979; 58: 497-9.

12. Schwartz AE, Navedo AT, Berman MF. Pancuronium increases the duration of electroencephalogram burst suppression in dogs anesthetized with isoflurane. Anesthesiology 1992; 77: 686-90.

13. Hodgson PS, Liu SS. Epidural lidocaine decreases sevoflurane requirement for adequate depth of anesthesia as measured by the Bispectral Index monitor. Anesthesiology 2001; 94: 799-803.

14. Kim KH, Choi YJ, Shim JH, Jeon WJ, Cho SY, Shin WJ, et al. Effect of nitrous oxide on bispectral index during sevoflurane anesthesia. Korean J Anesthesiol 2006; 50: 511-4.

15. Barr G, Jakobsson JG, Öwall A, Anderson RE. Nitrous oxide does not alter bispectral index: study with nitrous oxide as sole agent and as an adjunct to i.v. anaesthesia. Br J Anaesth 1999; 82: 827-30. 